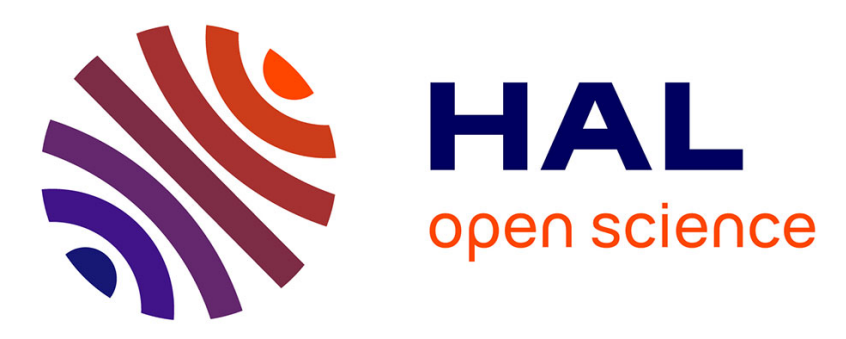

\title{
Ventcell conditions with mixed formulations for flow in porous media
}

Thi Thao Phuong Hoang, Caroline Japhet, Michel Kern, Jean E. Roberts

\section{To cite this version:}

Thi Thao Phuong Hoang, Caroline Japhet, Michel Kern, Jean E. Roberts. Ventcell conditions with mixed formulations for flow in porous media. 22th International conference on domain decomposition methods, Sep 2013, Lugano, Switzerland. hal-01113964

\section{HAL Id: hal-01113964 \\ https://inria.hal.science/hal-01113964}

Submitted on 6 Feb 2015

HAL is a multi-disciplinary open access archive for the deposit and dissemination of scientific research documents, whether they are published or not. The documents may come from teaching and research institutions in France or abroad, or from public or private research centers.
L'archive ouverte pluridisciplinaire HAL, est destinée au dépôt et à la diffusion de documents scientifiques de niveau recherche, publiés ou non, émanant des établissements d'enseignement et de recherche français ou étrangers, des laboratoires publics ou privés. 


\section{Ventcell conditions with mixed formulations for flow in porous media}

Thi Thao Phuong Hoang ${ }^{1}$, Caroline Japhet ${ }^{1,2}$, Michel Kern ${ }^{1}$, and Jean Roberts ${ }^{1}$

\section{Introduction}

The Optimized Schwarz method has been introduced and analyzed over the last decade, where the convergence speed of the Jacobi iteration is significantly enhanced by using general transmission conditions on the interfaces together with optimized parameters. In particular, Ventcell transmission conditions (see Japhet [1998], Japhet et al. [2001], Gander [2006], Dubois [2007], Japhet et al. [2014], Halpern and Hubert, Gander et al.) have been studied for the primal formulation with different numerical schemes showing that the convergence of the Optimized Schwarz algorithm with Ventcell conditions is improved over that with Robin conditions. Ventcell conditions are second order differential conditions, see Ventcel' [1959].

In this work, we study the Optimized Schwarz method with Ventcell conditions in the context of mixed formulations, which is not as straightforward as in the case of primal formulations and we have to introduce Lagrange multipliers on the interfaces to handle tangential derivatives involved in those conditions. Two dimensional numerical results for heterogeneous problems will be presented to compare the performance of the Ventcell transmission conditions with that of the Robin transmission conditions.

\section{A model problem and domain decomposition with Ventcell transmission conditions}

For an open, bounded domain $\Omega \subset \mathbb{R}^{d}(d=2,3)$ with Lipschitz boundary $\partial \Omega$, consider single phase flow in a porous medium written in mixed form:

$$
\begin{array}{rll}
\operatorname{div} \boldsymbol{u}=f & \text { in } \Omega, \\
\boldsymbol{K}^{-1} \boldsymbol{u}+\nabla p=0 & \text { in } \Omega, \\
p=0 & \text { on } \partial \Omega,
\end{array}
$$

where $p$ is the pressure, $\boldsymbol{u}$ the Darcy velocity, $f$ the source term and $\boldsymbol{K}$ a symmetric, positive definite, time independent, hydraulic conductivity (or permeability) tensor. For the sake of simplicity, we have imposed homogeneous Dirichlet condition on the boundary, other types of boundary conditions can

INRIA, Rocquencourt, France, Phuong.Hoang_Thi_Thao@inria.fr,Michel.Kern@inria.fr, Jean.Roberts@inria.fr .

Université Paris 13, LAGA, Villetaneuse, France, japhet@math.univ-paris13.fr

* This work was supported by ANDRA, the French Agency for Nuclear Waste Management. 
be treated similarly. The well-posedness of problem (1) is well-known (see, e.g., Brezzi [1974], Brezzi and Fortin [1991], Roberts and Thomas [1991]).

We consider a decomposition of $\Omega$ into two nonoverlapping subdomains, $\Omega_{1}$ and $\Omega_{2}$, separated by an interface $\Gamma$ :

$$
\Omega_{1} \cap \Omega_{2}=\emptyset ; \quad \Gamma=\partial \Omega_{1} \cap \partial \Omega_{2} \cap \Omega, \quad \Omega=\Omega_{1} \cup \Omega_{2} \cup \Gamma .
$$

Note that the same analysis can be extended to the case of many subdomains in bands. For $i=1,2$, let $\boldsymbol{n}_{i}$ denote the unit, outward pointing, normal on $\partial \Omega_{i}$, and for any scalar, vector or tensor valued function $\psi$ defined on $\Omega$, let $\psi_{i}$ denote the restriction of $\psi$ to $\Omega_{i}$. In order to write the Ventcell transmission conditions, we use the notation $\nabla_{\tau}$ and $\operatorname{div}_{\tau}$ for the tangential gradient and divergence operators on $\Gamma$ respectively. We denote by $\boldsymbol{K}_{i, \Gamma}$ the tangential component of the trace of $\boldsymbol{K}_{i}, i=1,2$, on $\Gamma$. A multidomain formulation equivalent to problem (1) is obtained by solving in each subdomain the following problem

$$
\begin{aligned}
\operatorname{div} \boldsymbol{u}_{i}=f & \text { in } \Omega_{i}, \\
\boldsymbol{K}_{i}^{-1} \boldsymbol{u}_{i}+\nabla p_{i}=0 & \text { in } \Omega_{i}, \\
p_{i}=0 & \text { on }\left(\partial \Omega_{i} \cap \partial \Omega\right),
\end{aligned}
$$

for $i=1,2$, together with the transmission conditions

$$
\begin{aligned}
& p_{1}=p_{2}, \\
& n_{1}+\boldsymbol{u}_{2} \cdot \boldsymbol{n}_{2}=0,
\end{aligned}
$$

Under sufficient regularity, one may replace (2) by optimized Ventcell transmission conditions, which were introduced and analyzed for primal formulations in Japhet [1998], Japhet et al. [2001]:

$$
\begin{gathered}
-\boldsymbol{u}_{1} \cdot \boldsymbol{n}_{1}+\alpha_{1,2} p_{1}+\beta_{1,2} \operatorname{div}_{\tau}\left(-\boldsymbol{K}_{2, \Gamma} \nabla_{\tau} p_{1}\right)=-\boldsymbol{u}_{2} \cdot \boldsymbol{n}_{1}+\alpha_{1,2} p_{2}+ \\
\beta_{1,2} \operatorname{div}_{\tau}\left(-\boldsymbol{K}_{2, \Gamma} \nabla_{\tau} p_{2}\right) \text { on } \Gamma, \\
-\boldsymbol{u}_{2} \cdot \boldsymbol{n}_{2}+\alpha_{2,1} p_{2}+\beta_{2,1} \operatorname{div}_{\tau}\left(-\boldsymbol{K}_{1, \Gamma} \nabla_{\tau} p_{2}\right)=-\boldsymbol{u}_{1} \cdot \boldsymbol{n}_{2}+\alpha_{2,1} p_{1}+ \\
\beta_{2,1} \operatorname{div}_{\tau}\left(-\boldsymbol{K}_{1, \Gamma} \nabla_{\tau} p_{1}\right) \text { on } \Gamma,
\end{gathered}
$$

where $\alpha_{i, j}$ and $\beta_{i, j}, i=1,2, j=(3-i)$, are positive constants. The conditions (3) are derived in such a way that they are equivalent to the original ones given in (2) (cf. references above). These parameters are chosen to optimize the convergence factor, see Japhet [1998], Japhet et al. [2001], Gander [2006], Dubois [2007].

\section{A multidomain formulation in mixed form}

In this section, we study Ventcell transmission conditions with mixed formulations. In order to handle second order terms involved in (3), we introduce Lagrange multipliers on the interface $\Gamma: p_{i, \Gamma}, i=1,2$, representing the pressure trace $p_{i}$ on $\Gamma$ and a vector field $\boldsymbol{u}_{\Gamma, i}:=-\boldsymbol{K}_{j, \Gamma} \nabla_{\tau} p_{i, \Gamma}, i=1,2, j=(3-i)$. We use the notation $\boldsymbol{u}_{\Gamma, i}$ instead of $\boldsymbol{u}_{i, \Gamma}$ to insist that $\boldsymbol{u}_{\Gamma, i}$ is not the tangential component of the trace of $\boldsymbol{u}_{i}$ on the interface. In fact $\boldsymbol{u}_{\Gamma, i}$ is used as an artificial tool for convergence purposes (it does not have a particular physical 
meaning). We rewrite (3) defined on $\Gamma$ as follows, for $i=1,2, j=(3-i)$ :

$$
\begin{gathered}
-\boldsymbol{u}_{i} \cdot \boldsymbol{n}_{i}+\alpha_{i, j} p_{i, \Gamma}+\beta_{i, j} \operatorname{div}_{\tau} \boldsymbol{u}_{\Gamma, i}=-\boldsymbol{u}_{j} \cdot \boldsymbol{n}_{i}+\alpha_{i, j} p_{j, \Gamma} \\
\quad+\beta_{i, j} \operatorname{div}_{\tau}\left(\boldsymbol{K}_{j, \Gamma} \boldsymbol{K}_{i, \Gamma}^{-1} \boldsymbol{u}_{\Gamma, j}\right), \\
\boldsymbol{K}_{j, \Gamma}^{-1} \boldsymbol{u}_{\Gamma, i}+\nabla_{\tau} p_{i, \Gamma}=0 .
\end{gathered}
$$

The corresponding multidomain problem consists of solving in the subdomains the problem, for $i=1,2, j=(3-i)$ :

$$
\begin{aligned}
\operatorname{div} \boldsymbol{u}_{i} & =f & & \text { in } \Omega_{i}, \\
\boldsymbol{K}_{i}^{-1} \boldsymbol{u}_{i}+\nabla p_{i} & =0 & & \text { in } \Omega_{i}, \\
p_{i} & =0 & & \text { on }\left(\partial \Omega_{i} \cap \partial \Omega\right), \\
-\boldsymbol{u}_{i} \cdot \boldsymbol{n}_{i}+\alpha_{i, j} p_{i, \Gamma}+\beta_{i, j} \operatorname{div}_{\tau} \boldsymbol{u}_{\Gamma, i} & =-\boldsymbol{u}_{j} \cdot \boldsymbol{n}_{i}+\alpha_{i, j} p_{j, \Gamma} & & \\
+\beta_{i, j} & \operatorname{div}_{\tau}\left(\boldsymbol{K}_{j, \Gamma} \boldsymbol{K}_{i, \Gamma}^{-1} \boldsymbol{u}_{\Gamma, j}\right) & & \text { on } \Gamma, \\
\boldsymbol{K}_{j, \Gamma}^{-1} \boldsymbol{u}_{\Gamma, i}+\nabla_{\tau} p_{i, \Gamma} & =0 & & \text { on } \Gamma, \\
p_{i, \Gamma} & =0 & & \text { on } \partial \Gamma .
\end{aligned}
$$

This can be seen as a coupling problem between a $d$-dimensional PDE in the subdomain $\Omega_{i}$ and a $(d-1)$-dimensional PDE on the interface $\Gamma$, and both PDEs are written in mixed form. Under a suitable regularity hypothesis the multidomain problem (5) is equivalent to the monodomain problem (1). Details of the proof can be found in [Hoang, 2013, pp. $94-95]$.

\subsection{Well-posedness of the Ventcell local problem}

For an open, bounded domain $\mathcal{O} \subset \mathbb{R}^{d}(d=2,3)$ with Lipschitz boundary $\partial \mathcal{O}$, consider the following elliptic problem written in mixed form with Ventcell boundary conditions

$$
\begin{aligned}
\operatorname{div} \boldsymbol{u}_{\mathcal{O}} & =f_{\mathcal{O}} & & \text { in } \mathcal{O} \\
\boldsymbol{K}^{-1} \boldsymbol{u}_{\mathcal{O}}+\nabla p_{\mathcal{O}} & =0 & & \text { in } \mathcal{O} \\
-\boldsymbol{u}_{\mathcal{O}} \cdot n+\alpha p_{\partial \mathcal{O}}+\beta \operatorname{div}_{\tau} \tilde{\boldsymbol{u}}_{\partial \mathcal{O}} & =f_{\partial \mathcal{O}} & & \text { on } \partial \mathcal{O} \\
\tilde{\boldsymbol{K}}_{\partial \mathcal{O}}^{-1} \tilde{\boldsymbol{u}}_{\partial \mathcal{O}}+\nabla_{\tau} p_{\partial \mathcal{O}} & =0 & & \text { on } \partial \mathcal{O}
\end{aligned}
$$

where $n$ is the unit, outward pointing, normal vector on $\partial \mathcal{O}, K(\cdot) \in \mathbb{R}^{d^{2}}$ and $\tilde{\boldsymbol{K}}_{\partial \mathcal{O}}(\cdot) \in \mathbb{R}^{(d-1)^{2}}$ are given, and $\alpha$ and $\beta$ are positive constants.

In order to write the weak formulation of problem (6), we define the following Hilbert spaces

$$
\begin{aligned}
& M=\left\{\mu=\left(\mu_{\mathcal{O}}, \mu_{\partial \mathcal{O}}\right) \in L^{2}(\mathcal{O}) \times L^{2}(\partial \mathcal{O})\right\} \\
& \Sigma=\left\{\boldsymbol{v}=\left(\boldsymbol{v}_{\mathcal{O}}, \tilde{\boldsymbol{v}}_{\partial \mathcal{O}}\right) \in \boldsymbol{L}^{\mathbf{2}}(\mathcal{O}) \times \boldsymbol{L}^{\mathbf{2}}(\partial \mathcal{O}): \operatorname{div} \boldsymbol{v}_{\mathcal{O}} \in L^{2}(\mathcal{O})\right. \text { and } \\
&\left.\quad \beta \operatorname{div}_{\tau} \tilde{\boldsymbol{v}}_{\partial \mathcal{O}}-\boldsymbol{v}_{\mathcal{O}} \cdot \boldsymbol{n}_{\mid \partial \mathcal{O}} \in L^{2}(\partial \mathcal{O})\right\}
\end{aligned}
$$

equipped with the norms

$$
\begin{aligned}
\|\mu\|_{M}^{2} & =\left\|\mu_{\mathcal{O}}\right\|_{\mathcal{O}}^{2}+\left\|\mu_{\partial \mathcal{O}}\right\|_{\partial \mathcal{O}}^{2} \\
\|\boldsymbol{v}\|_{\Sigma}^{2} & =\left\|\boldsymbol{v}_{\mathcal{O}}\right\|_{\mathcal{O}}^{2}+\left\|\operatorname{div} \boldsymbol{v}_{\mathcal{O}}\right\|_{\mathcal{O}}^{2}+\left\|\tilde{\boldsymbol{v}}_{\partial \mathcal{O}}\right\|_{\partial \mathcal{O}}^{2}+\left\|\beta \operatorname{div} \tilde{\boldsymbol{v}}_{\partial \mathcal{O}}-\boldsymbol{v}_{\mathcal{O}} \cdot \boldsymbol{n}_{\mid \partial \mathcal{O}}\right\|_{\partial \mathcal{O}}^{2}
\end{aligned}
$$


where $\|\cdot\|_{\mathcal{O}}$ and $\|\cdot\|_{\partial \mathcal{O}}$ are the $L^{2}(\mathcal{O})$ and $L^{2}(\partial \mathcal{O})-$ norms, respectively. We denote by $(\cdot, \cdot)_{\mathcal{O}}$ and $(\cdot, \cdot)_{\partial \mathcal{O}}$ the inner products of $L^{2}(\mathcal{O})$ and $L^{2}(\partial \mathcal{O})$.

Next, define the following bilinear forms (recall that $\beta$ is a positive constant) on $\Sigma \times \Sigma, \Sigma \times M$ and $M \times M$ respectively:

$$
\begin{aligned}
& a(\boldsymbol{u}, \boldsymbol{v})=\left(\boldsymbol{K}^{-1} \boldsymbol{u}_{\mathcal{O}}, \boldsymbol{v}_{\mathcal{O}}\right)_{\mathcal{O}}+\left(\beta \tilde{\boldsymbol{K}}_{\partial \mathcal{O}}^{-1} \tilde{\boldsymbol{u}}_{\partial \mathcal{O}}, \tilde{\boldsymbol{v}}_{\partial \mathcal{O}}\right)_{\partial \mathcal{O}}, \\
& b(\boldsymbol{u}, \mu)=\left(\operatorname{div} \boldsymbol{u}_{\mathcal{O}}, \mu_{\mathcal{O}}\right)_{\mathcal{O}}+\left(\beta \operatorname{div}_{\tau} \tilde{\boldsymbol{u}}_{\partial \mathcal{O}}-\boldsymbol{u}_{\mathcal{O}} \cdot \boldsymbol{n}_{\mid \partial \mathcal{O}}, \mu_{\partial \mathcal{O}}\right)_{\partial \mathcal{O}}, \\
& c(p, \mu)=\left(\alpha p_{\partial \mathcal{O}}, \mu_{\partial \mathcal{O}}\right)_{\partial \mathcal{O}},
\end{aligned}
$$

and the linear form defined on $M$ by:

$$
L_{f}(\mu)=\left(f_{\mathcal{O}}, \mu_{\mathcal{O}}\right)_{\mathcal{O}}+\left(f_{\partial \mathcal{O}}, \mu_{\partial \mathcal{O}}\right)_{\partial \mathcal{O}} .
$$

With these spaces and forms, the weak form of (6) can be written as follows:

Find $(p, \boldsymbol{u}) \in M \times \Sigma$ such that

$$
\begin{array}{rlrl}
a(\boldsymbol{u}, \boldsymbol{v})-b(\boldsymbol{v}, p) & =0 & \forall \boldsymbol{v} & \in \Sigma, \\
-b(\boldsymbol{u}, \mu)-c(p, \mu) & =-L_{f}(\mu) \forall \mu & \in M .
\end{array}
$$

Theorem 1. Assume that there exist positive constants $K_{-}$and $K_{+}$such that $\varsigma^{T} \boldsymbol{K}^{-1}(\cdot) \varsigma \geq K_{-}|\varsigma|^{2}$, and $|\boldsymbol{K}(\cdot) \varsigma| \leq K_{+}|\varsigma|$ a.e. in $\mathcal{O}$ and $\forall \varsigma \in \mathbb{R}^{d}$; and that $\eta^{T} \tilde{\boldsymbol{K}}_{\partial \mathcal{O}}^{-1}(\cdot) \eta \geq K_{-}|\eta|^{2}$, and $\left|\tilde{\boldsymbol{K}}_{\partial \mathcal{O}}(\cdot) \eta\right| \leq K_{+}|\eta|$ a.e. in $\partial \mathcal{O}$ and $\forall \eta \in$ $\mathbb{R}^{d-1}$. If $\left(f_{\mathcal{O}}, f_{\partial \mathcal{O}}\right)$ is in $M$ then there exists a unique solution $(p, \boldsymbol{u}) \in M \times \Sigma$ of problem (7).

Proof. The existence and uniqueness of the solution of (7) is a generalization of the classical case (see [Brezzi and Fortin, 1991, pp. 47 - 50], [Roberts and Thomas, 1991, pp. 572 - 573]). See [Hoang, 2013, pp. 96 - 98] for details of the proof of Theorem 1 .

\subsection{An interface problem}

In this subsection, we derive an interface problem associated with the multidomain problem (5). With this aim, we define the Ventcell-to-Ventcell operator $\mathcal{S}_{i}^{\mathrm{VtV}}$ (note that we have assumed sufficient regularity of the solution as in Section 2), which depends on the parameters $\alpha_{i, j}$ and $\beta_{i, j}$, for $i=1,2$, and $j=(3-i)$, as follows

$$
\begin{gathered}
\mathcal{S}_{i}^{\mathrm{VtV}}: L^{2}(\Gamma) \times L^{2}\left(\Omega_{i}\right) \rightarrow L^{2}(\Gamma) \\
(\vartheta, f) \longmapsto \mathcal{S}_{i}^{\mathrm{VtV}}(\vartheta, f)=-\boldsymbol{u}_{i} \cdot \boldsymbol{n}_{j \mid \Gamma}+\alpha_{j, i} p_{i, \Gamma}+\beta_{j, i} \operatorname{div}_{\tau}\left(\boldsymbol{K}_{i, \Gamma} \boldsymbol{K}_{j, \Gamma}^{-1} \boldsymbol{u}_{\Gamma, i}\right),
\end{gathered}
$$

where $\left(p_{i}, \boldsymbol{u}_{i}, p_{i, \Gamma}, \boldsymbol{u}_{\Gamma, i}\right), i=1,2$, is the solution of

$$
\begin{aligned}
& \operatorname{div} \boldsymbol{u}_{i}=f \text { in } \Omega_{i}, \\
& \boldsymbol{K}_{i}^{-1} \boldsymbol{u}_{i}+\nabla p_{i}=0 \text { in } \Omega_{i} \\
& p_{i}=0 \text { on }\left(\partial \Omega_{i} \cap \partial \Omega\right) \text {, } \\
& -\boldsymbol{u}_{i} \cdot \boldsymbol{n}_{i}+\alpha_{i, j} p_{i, \Gamma}+\beta_{i, j} \operatorname{div}_{\tau} \boldsymbol{u}_{\Gamma, i}=\vartheta \quad \text { on } \Gamma, \\
& \boldsymbol{K}_{j, \Gamma}^{-1} \boldsymbol{u}_{\Gamma, i}+\nabla_{\tau} p_{i, \Gamma}=0 \quad \text { on } \Gamma \\
& p_{i, \Gamma}=0 \text { on } \partial \Gamma \text {. }
\end{aligned}
$$


The well-posedness of problem (8) is given by an extension of Theorem 1 . The interface problem, corresponding to the Ventcell transmission conditions (4), is defined by

$$
\begin{aligned}
& \vartheta_{1}=\mathcal{S}_{2}^{\mathrm{VtV}}\left(\vartheta_{2}, f\right) \\
& \vartheta_{2}=\mathcal{S}_{1}^{\mathrm{VtV}}\left(\vartheta_{1}, f\right)
\end{aligned} \quad \text { on } \Gamma
$$

or equivalently,

$$
\mathcal{S}_{\boldsymbol{V}}\left(\begin{array}{l}
\vartheta_{1} \\
\vartheta_{2}
\end{array}\right)=\chi_{\boldsymbol{V}}(f), \quad \text { on } \Gamma
$$

where

$$
\begin{aligned}
& \mathcal{S}_{\boldsymbol{V}}: L^{2}(\Gamma) \times L^{2}(\Gamma) \longrightarrow \quad L^{2}(\Gamma) \times L^{2}(\Gamma) \\
& \left(\begin{array}{l}
\vartheta_{1} \\
\vartheta_{2}
\end{array}\right) \quad \longmapsto\left(\begin{array}{l}
\vartheta_{1}-\mathcal{S}_{2}^{\mathrm{VtV}}\left(\vartheta_{2}, 0\right) \\
\vartheta_{2}-\mathcal{S}_{1}^{\mathrm{VtV}}\left(\vartheta_{1}, 0\right)
\end{array}\right),
\end{aligned}
$$

and

$$
\begin{aligned}
& \chi_{\boldsymbol{V}}: L^{2}(\Gamma) \longrightarrow L^{2}(\Gamma) \times L^{2}(\Gamma) \\
& f \quad \longmapsto\left(\begin{array}{l}
\mathcal{S}_{2}^{\mathrm{VtV}}(0, f) \\
\mathcal{S}_{1}^{\mathrm{VtV}}(0, f)
\end{array}\right) .
\end{aligned}
$$

One can solve problem (10) iteratively using Jacobi iteration or a Krylov method (e.g. GMRES, see for example Japhet et al. [2001]) the right hand side is computed (only once) by solving problem (8) in each subdomain with $\vartheta=0$; then for a given pair of vectors $\left(\vartheta_{1}, \vartheta_{2}\right)$, the matrix vector product is obtained (at each iteration) by solving, for $i=1,2$, subdomain problem (8) in $\Omega_{i}$ with $\vartheta=\vartheta_{i}$ and with $f=0$. If one uses Jacobi iteration for solving (10), the resulting algorithm is equivalent to the optimized Schwarz algorithm with Ventcell transmission conditions (see Japhet [1998], Gander [2006]).

\section{Numerical results}

We consider a domain $\Omega=(0, \pi)^{2}$ and its decomposition into two nonoverlapping subdomains $\Omega_{1}=\left(0, \frac{\pi}{2}\right) \times(0, \pi)$ and $\Omega_{2}=\left(\frac{\pi}{2}, \pi\right) \times(0, \pi)$. The permeability is $\boldsymbol{K}=\mathfrak{K} \boldsymbol{I}$ isotropic and constant on each subdomain, where $\boldsymbol{I}$ is the $2 \mathrm{D}$ identity matrix. We take $\mathfrak{K}_{1}=1 / \mathcal{K}$ and $\mathfrak{K}_{2}=1$, where $\mathcal{K}=1,10$ or 100. The exact solution is $p(x, y)=\cos (\pi x) \sin (\pi y)$. For the spatial discretization, we use mixed finite elements (with interface Lagrange multipliers) with the lowest order Raviart-Thomas spaces on rectangles (see Brezzi and Fortin [1991], Roberts and Thomas [1991]).

Remark 1. In order to handle the discontinuous coefficients, we use the optimized, weighted Ventcell parameters defined by

$$
\begin{aligned}
\alpha_{1,2} & =\mathfrak{K}_{2} \alpha, \quad \alpha_{2,1}=\mathfrak{K}_{1} \alpha, \\
\beta_{1,2} & =\mathfrak{K}_{2} \beta, \quad \beta_{2,1}=\mathfrak{K}_{1} \beta .
\end{aligned}
$$

The calculation of these parameters is done as in Dubois [2007].

We first study the convergence behavior of the optimized Schwarz method with the optimized weighted Ventcell parameters. To that purpose, we con- 
sider the error equation, i.e. with $f=0$ and homogeneous Dirichlet boundary conditions. We start with a random initial guess on the interface and compute the error in the $L^{2}(\Omega)$-norm of the pressure $p$ and of the velocity $\boldsymbol{u}$. Table 1 gives the number of iterations needed to reach an error reduction of $10^{-6}$ first in $p$ and then in $u$ (in square brackets) as the mesh is refined. Both GMRES and Jacobi iterations are considered. For homogeneous case $(\mathcal{K}=1)$, GMRES significantly improves the convergence speed (by a factor of 2) and also the asymptotic results compared to the Jacobi iteration. These results are consistent with those obtained with primal formulations in Gander [2006] (where a finite difference scheme is used). As the ratio $\mathcal{K}$ increases, the number of iterations is smaller and GMRES does not greatly accelerate the convergence speed compared to Jacobi iterations. Also for large values of the contrast $\mathcal{K}$, the convergence rate of the algorithms with GMRES or Jacobi are almost independent of the mesh size (since the optimized parameters play the role of a preconditioner). This is also the case where a primal formulation and a finite volume method are used (cf. Dubois [2007]).

\begin{tabular}{|l|c|c|c|c|c|c|}
\hline \multirow{2}{*}{$h$} & \multicolumn{2}{|c|}{$\mathcal{K}=1$} & \multicolumn{2}{c|}{$\mathcal{K}=10$} & \multicolumn{2}{c|}{$\mathcal{K}=100$} \\
\cline { 2 - 7 } & Jacobi & GMRES & Jacobi & GMRES & Jacobi & GMRES \\
\hline$\pi / 50$ & $15[15]$ & $10[11]$ & $11[10]$ & $9[9]$ & $7[6]$ & $7[7]$ \\
\hline$\pi / 100$ & $17[18]$ & $11[12]$ & $11[10]$ & $9[9]$ & $7[6]$ & $7[7]$ \\
\hline$\pi / 200$ & $21[21]$ & $13[13]$ & $11[10]$ & $9[9]$ & $7[6]$ & $7[7]$ \\
\hline$\pi / 400$ & $25[25]$ & $14[14]$ & $11[10]$ & $10[9]$ & $7[6]$ & $8[8]$ \\
\hline$\pi / 800$ & $29[29]$ & $15[16]$ & $13[12]$ & $10[10]$ & $7[6]$ & $8[8]$ \\
\hline
\end{tabular}

Table 1 Number of iterations required to reach an error reduction of $10^{-6}$ in $p$ and in $\boldsymbol{u}$ (in square brackets) for different permeability ratios, and for different values of the discretization parameter $h$.

Next we verify the performance of the optimized parameters, computed by numerically minimizing the continuous convergence factor. We take $h=\pi / 100$, vary $\alpha$ and $\beta$, and compute the error in the velocity $\boldsymbol{u}$ after a fixed number of Jacobi iterations for different permeability ratios. The results are shown in Figure 1 for $\mathcal{K}=1$ (\# iter $=20$ iterations), $\mathcal{K}=10$ (\# iter $=12$ iterations) and $\mathcal{K}=100$ (\# iter $=8$ iterations) respectively. We see that, in all cases, the optimized weighted Ventcell parameters (the red star) are located close to those giving the smallest error after the same number of iterations.

Finally, we illustrate the improvement obtained using Ventcell transmission conditions over the Robin conditions (i.e. $\beta=0$ ).

We consider the optimized 2-sided Robin parameters with $\alpha_{1,2} \neq \alpha_{2,1}$ and $\beta_{1,2}=\beta_{2,1}=0$. Figures 2 shows the error in the pressure versus the number of iterations using Jacobi (on the left) and GMRES (on the right) for different diffusion ratios, $\mathcal{K}=1$ and $\mathcal{K}=100$ respectively.

We see that for the homogeneous case $(\mathcal{K}=1)$, with Jacobi iterations the optimized weighted Ventcell converges significantly faster than the optimized 2 -sided Robin (by a factor of 2). As $\mathcal{K}$ increases, the optimized weighted Ventcell and the optimized 2-sided Robin become comparable. With GMRES, the difference in the convergence of the two types of optimized parameters 

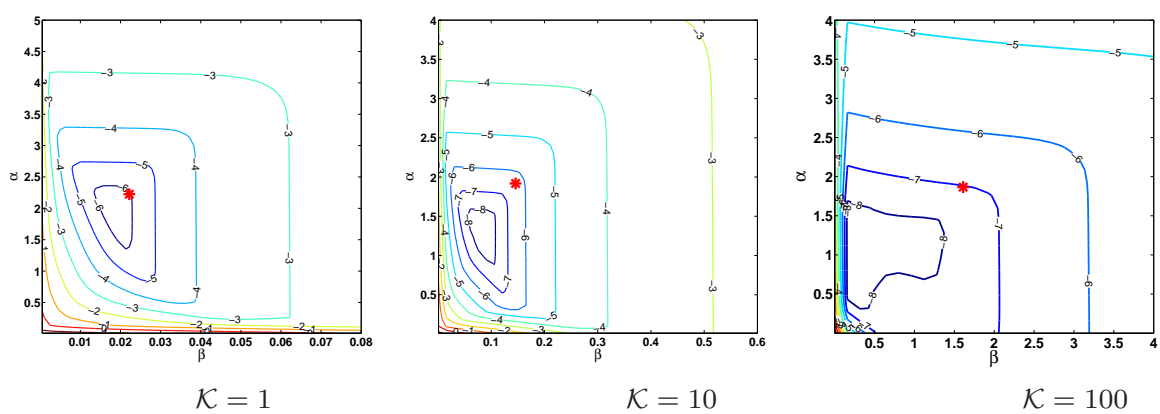

Fig. 1 Level curves for the error in the velocity (in logarithmic scale) after some fixed number of Jacobi iterations for various values of the parameters $\alpha$ and $\beta$ and for different permeability ratios $\mathcal{K}$. The red star shows the optimized parameters.
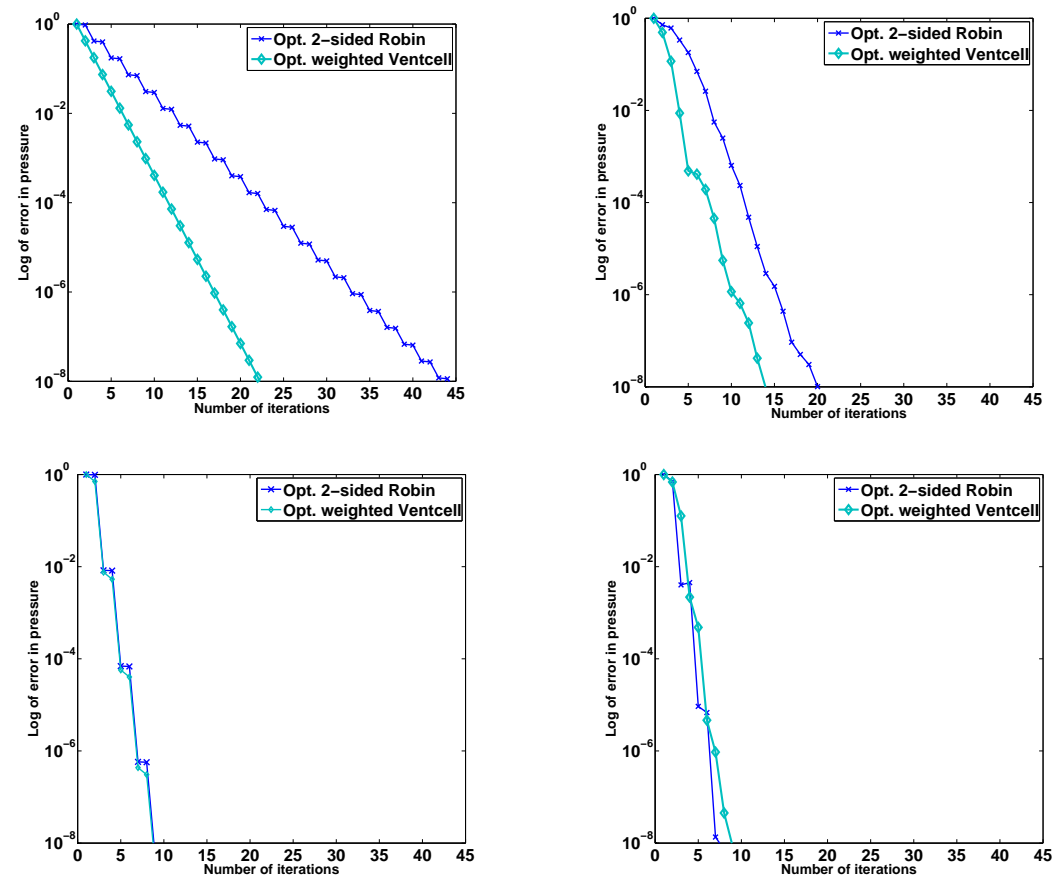

Fig. $2 L^{2}$ error in the pressure $p$ for $\mathcal{K}=1$ (top) and $\mathcal{K}=100$ (bottom): Jacobi (left) and GMRES (right).

becomes less significant, especially for high diffusion ratios. These results are for a symmetric two subdomain case with a conforming mesh, Ventcell transmission conditions may have a more important effect on the convergence (compared with Robin transmission conditions) when many subdomains and nonmatching grids are considered (cf. Japhet et al. [2014]). 


\section{References}

F. Brezzi. On the existence, uniqueness and approximation of saddle-point problems arising from Lagrangian multipliers. RAIRO Anal. Numér., 8: 129-151, 1974.

F. Brezzi and M. Fortin. Mixed and hybrid finite elements methods. SpringerVerlag, New York, 1991. ISBN 3540975829.

O. Dubois. Optimized Schwarz methods for the advection-diffusion equation and for problems with discontinuous coefficients. PhD thesis, McGill University, 2007.

M. J. Gander. Optimized Schwarz methods. SIAM J. Numer. Anal., 44(2): 699-731, 2006.

M. J. Gander, L. Halpern, F. Hubert, and S. Krell. DDFV Ventcell Schwarz algorithms. In Proceedings of the 22nd International Conference on Domain Decomposition Methods.

L. Halpern and F. Hubert. Finite volume Ventcell-Schwarz algorithm for advection-diffusion equations. SIAM J. Numer. Anal., (to appear).

T.T.P Hoang. Space-time domain decomposition methods for mixed formulations of flow and transport problems in porous media. $\mathrm{PhD}$ thesis, University Paris 6, 2013.

C. Japhet. Optimized Krylov-Ventcell method. Application to convectiondiffusion problems. In U. Bjørstad, M. Espedal, and D.E. Keyes, editors, Domain decomposition methods in science and engineering IX, pages $382-$ 389. John Wiley \& Sons Ltd, 1998.

C. Japhet, F. Nataf, and F. Rogier. The Optimized Order 2 method. application to convection-diffusion problems. Future Gener. Comp. Sy., 18(1): 17-30, 2001.

C. Japhet, Y. Maday, and F. Nataf. A new interface cement equilibrated mortar method with Ventcel conditions. In J. Erhel, M.J. Gander, L. Halpern, G. Pichot, T. Sassi, and O. Widlund, editors, Domain decomposition methods in science and engineering XXI, Lecture Notes in Computational Science and Engineering, volume 98, pages 329-336. Springer, 2014.

J. E. Roberts and J.-M. Thomas. Mixed and hybrid methods. In Handbook of numerical analysis, Vol. II, Handb. Numer. Anal., II, pages 523-639. North-Holland, Amsterdam, 1991.

A. D. Ventcel'. On boundary conditions for multi-dimensional diffusion processes. Theor. Probability Appl., 4:164177, 1959. 